\title{
Overview of common errors and pitfalls to avoid in the acquisition and interpretation of ultrasound imaging of the abdominal aorta
}

This article was published in the following Dove Press journal:

Journal of Vascular Diagnostics and Interventions

9 October 2017

Number of times this article has been viewed

\section{Penelope C Lema \\ Janice H Kim \\ Erika St James}

Department of Emergency Medicine, University at Buffalo Jacobs School of Medicine and Biomedical Sciences, Buffalo, NY, USA
Correspondence: Penelope C Lema Department of Emergency Medicine, Buffalo General Hospital, 100 High Street, D-6, Buffalo, NY 14203, USA

$\mathrm{Tel}+\mathrm{I} 7168592460$

$\mathrm{Fax}+\mathrm{I} 7168591555$

Email Penelope.chun@gmail.com
Abstract: Abdominal aortic aneurysms (AAAs) occur when the aorta diameter is $>3 \mathrm{~cm}$ (30 $\mathrm{mm}$ ). Ruptured AAA has a high mortality rate. Ultrasound is the imaging modality of choice to screen for this vascular disease. There are many common errors and pitfalls in the acquisition and interpretation of ultrasound imaging of the abdominal aorta, such as measurement errors and variations in technique, misdiagnosis errors, difficulty with visualization of the aorta and a wide range of sonographer experience. We review the common errors and pitfalls to recognize and avoid in ultrasound imaging of the abdominal aorta.

Keywords: ultrasound, abdominal aortic aneurysm, AAA, pitfalls and errors, imaging technique

\section{Introduction}

Vascular aneurysms are defined by a 1.5 increase from baseline luminal diameter. Given that the average abdominal aorta measures $2 \mathrm{~cm}$, an abdominal aortic aneurysm (AAA) is defined by a measurement of $3 \mathrm{~cm}(30 \mathrm{~mm})$ or greater. ${ }^{1}$ The prevalence of AAA increases with age; also, it is higher in males and in those with a smoking history, atherosclerotic disease and family history. ${ }^{2} \mathrm{~A}$ feared complication of AAA is rupture. Its overall prehospital mortality reaches $85 \%-90 \%$ and in-hospital mortality approaches $50 \% .^{3}$ AAA rupture is directly correlated to its diameter. The annual risk of rupture is $<0.5 \%$ for those measuring $<4 \mathrm{~cm}, 0.5 \%-5 \%$ per year for $4-5 \mathrm{~cm}, 3 \%-15 \%$ for $5-6$ $\mathrm{cm}, 10 \%-20 \%$ for $6-7 \mathrm{~cm}, 20 \%-40 \%$ for $7-8 \mathrm{~cm}$ and $30 \%-50 \%$ per year for those measuring $>8 \mathrm{~cm} .{ }^{4}$ Those with predisposing factors should be screened for this disease. ${ }^{5}$

Ruptured AAA is associated with high mortality if not rapidly diagnosed and surgically repaired. Point-of-care ultrasonography in the emergency setting can be life-saving. Previous studies have reported sensitivities of $97.7 \%-100 \%$ and specificities of $94.1 \%-100 \%$ in the ability to diagnose AAA with ultrasound. ${ }^{6-8}$ Ultrasonography is portable, costeffective, lacks ionizing radiation and is the image modality of choice to screen for this disease. ${ }^{5}$ Multiple sonographic views of the abdominal aorta should be obtained when feasible. Visualization of the proximal, mid and distal abdominal aorta, including the iliac artery bifurcation, is done in the longitudinal and axial planes (Figure 1A, B). ${ }^{1}$ In order to accurately assess AAAs, the sonographer should be cognizant of the common errors and pitfalls to be avoided in the acquisition and interpretation of vascular ultrasound imaging.

\section{Measurement errors}

AAA screening programs in the UK and USA allow for $<5 \mathrm{~mm}$ discrepancy in measurement of the aorta diameter. ${ }^{9}$ The aorta diameter may be slightly greater when 
measured with ultrasound compared to that measured with computed tomography (CT). ${ }^{10}$ Ultrasound is highly operator dependent, and inter- and intraobserver variability does exist. $^{9-14}$ The aorta diameter measured in the anteroposterior (AP) plane is reproduced more accurately compared to that measured in the transverse (TR) plane. ${ }^{10}$

Three approaches exist for measurement of the aorta diameter with ultrasound: inner-to-inner (ITI), leading-edge-toleading edge and outer-to-outer (OTO), as shown in Figure 2. ${ }^{9}$ In the ITI approach, the calipers of the aorta diameter are measured from the anterior inner lumen of the aorta wall to the posterior inner lumen of the aorta wall. In the leadingedge-to-leading edge approach, the calipers are placed on the outer layer of the anterior aorta wall and measured to the inner lumen of the posterior wall. In the OTO approach, the calipers are placed from the outer anterior aorta wall to the outer posterior aorta wall. ${ }^{11}$ The ITI and OTO are the two most commonly used methods. The UK's AAA screening program, The NHS Abdominal Aortic Aneurysm Screening Programme, is a randomized AAA screening program that uses the ITI technique to diagnose aneurysms based on evidence from the Multicentre Aneurysm Screening Study. ${ }^{15,16}$
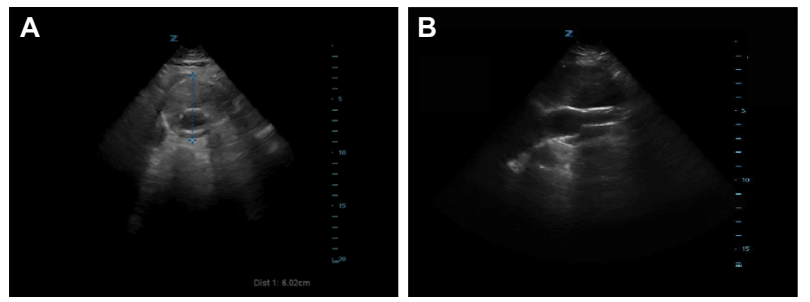

Figure I (A) Axial and (B) sagittal ultrasound images of a $6 \mathrm{~cm}$ abdominal aortic aneurysm with endovascular stent.
Improved inter- and intrarater reliability and repeatability has been demonstrated with the ITI approach. ${ }^{12}$ Proponents of the ITI approach suggest that there is better resolution at the inner wall of the posterior aorta compared to the outer wall of the aorta where the adventitia blends into the surrounding connective tissue. ${ }^{17}$ This subsequently produces weak reflective boundaries at the outer wall of the aorta. ${ }^{18}$ There are ramifications if the ITI approach is solely used. Patients with lower aorta measurements may miss the cutoff for AAA screening programs. Some have even advocated for lowering the threshold for entry into AAA surveillance programs to 26 $\mathrm{mm}$ with the ITI approach. ${ }^{19}$ Vascular surgeons and national intervention criteria, however, may base intervention on the OTO aorta diameter, which has caused confusion with interpretations of the ITI versus OTO techniques. ${ }^{13,17,20}$ The Screening Abdominal Aortic Aneurysm Very Efficiently program in the USA refers to the OTO technique to screen for aneurysms. ${ }^{9,21}$ The American Institute of Ultrasound in Medicine and the American College of Emergency Physicians both recommend the OTO method. The American College of Emergency Physicians further recommends that both the AP and TR (side-to-side) aorta diameters should be measured in the axial planes when feasible. The anterior and posterior aorta walls are more precise and sharply defined. However, measurement of the lateral walls may improve aneurysm assessment, since many AAAs have larger side-to-side diameters compared to the AP diameter. ${ }^{1,22}$

A mural thrombus is frequently present within an AAA. ${ }^{23}$ The OTO approach may prevent a false-negative measurement if the aorta lumen is inadvertently measured rather than the entire AP diameter of the aneurysmal aorta (Figure 3).

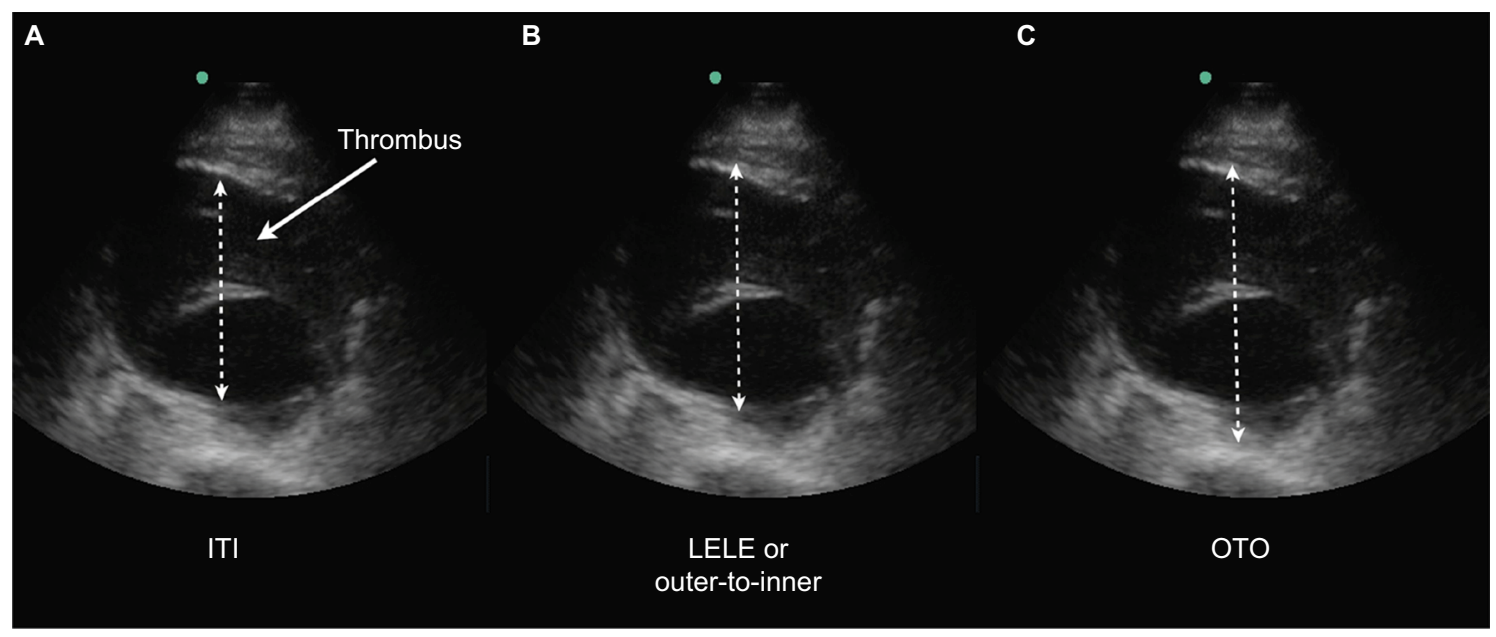

Figure 2 Axial ultrasound image of an abdominal aortic aneurysm with thrombus, demonstrating the three common methods to measure the aorta AP diameter. Notes: (A) ITI, (B) LELE and (C) OTO.

Abbreviations: AP, anteroposterior; ITI, inner-to-inner; LELE, leading edge-to-leading edge; OTO, outer-to-outer. 


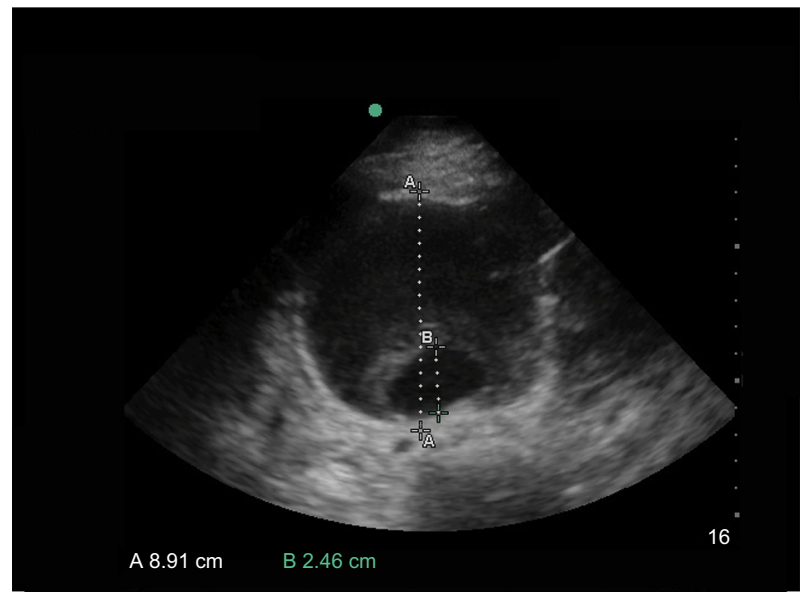

Figure 3 Ultrasound of an AAA in the axial plane.

Notes: (A) The OTO AP measurement correctly identifies the $8.9 \mathrm{~cm}$ AAA. (B) The ITI measurement may inadvertently underestimate an aneurysm and mural thrombus.

Abbreviations: AAA, abdominal aortic aneurysm; AP, anteroposterior; ITI, innerto-inner; ОTO, outer-to-outer.

Occasionally, the thrombus can be mistaken as the aorta wall. Visualization of the thrombus in TR and longitudinal views can aid in evaluation of the aneurysm and thrombus. Identification of the clot and differentiation of the aorta wall are paramount for an accurate measurement of AAA diameter. ${ }^{24}$

The morphology of the aorta can vary. The abdominal aorta is occasionally tortuous, and sonographic measurements of the aorta diameter may be inaccurate depending on the trajectory of the aorta and the location of measurement. The exact axial plane of the abdominal aorta may be difficult to isolate with a tortuous aorta. Oblique or angulated measurement of the aorta diameter can lead to an overestimation of the aorta diameter. Occasionally, the cross section of the aorta appears elliptical, which will result in inaccurate measurements of the aorta diameter. ${ }^{25}$ Sonographers should ensure that the ultrasound beam is in plane with the axis of the aorta, instead of being at an angle. A thorough sonographic evaluation of the entire length of the abdominal aorta from cephalad to caudad will identify the trajectory of the aorta and detect the presence of fusiform or saccular aneurysms.

Fusiform aneurysms involve the entire circumference of the aorta wall and appear concentrically dilated. Saccular aneurysms involve a focal eccentric outpouching of the aorta wall. ${ }^{26,27}$ A large majority of the aneurysms are fusiform and are often associated with arterial wall degeneration secondary to atherosclerotic disease. ${ }^{28}$ While the etiology of saccular aneurysms remains primarily associated with atherosclerosis, occasionally, there are other independent causes of their formation as well, such as infection and inflammation, trauma, aortic ulcer or previous aorta surgery. ${ }^{28,29}$ The natural progression and the risk of rupture of saccular aneurysms are still unknown due to their varied etiology. It is believed that saccular aneurysms historically have a greater risk of rupture compared to fusiform aneurysms and are often repaired at smaller diameters. ${ }^{28-30}$ Even though both men and women have suffered aortic ruptures while under surveillance, women have been found to rupture at smaller aorta diameters in comparison to men. ${ }^{31,32}$ Sonographic evaluation of the aorta in two planes can prevent inadvertent missed saccular aneurysms. Once a thorough ultrasound evaluation of the entire length of the abdominal aorta is completed in the axial (TR) plane from cephalad to caudad, an evaluation of the aorta should be performed in the sagittal (longitudinal) plane for the presence of focal saccular aneurysms.

Operator error can occur if the midline of the aorta is not properly imaged in the sagittal view. The cylinder tangent effect occurs if the plane of the beam enters the aorta at a tangent and a falsely reduced AP diameter is resulted (Figure 4). ${ }^{26}$ Avoid this error by imaging the aorta in the axial plane and then slowly rotating the transducer until the sagittal plane of the aorta is visualized.

\section{Misdiagnosis errors}

In the longitudinal view, the inferior vena cava (IVC) can be misidentified as the abdominal aorta if the sonographer sweeps too far to the patient's right in the parasagittal plane, since the IVC and aorta travel parallel to each other. ${ }^{26} \mathrm{~A}$ few distinctive features can help differentiate the IVC from the aorta. The IVC appears thin walled and is compressible, compared to the thick-walled and pulsatile aorta. The celiac trunk and the superior mesenteric artery branch off the aorta and can be visualized in the sagittal plane to confirm that the aorta is being visualized rather than the IVC. ${ }^{26}$ Again, imaging the aorta in two planes may prevent errors.
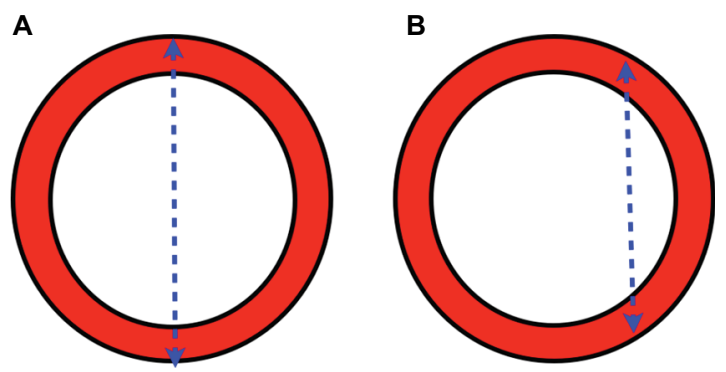

Figure 4 The cylinder tangent effect.

Note: (A) A longitudinal beam through the center of the aorta will provide the maximal AP diameter, whereas $(\mathbf{B})$ a beam off to the side or tangent will result in a falsely smaller diameter.

Abbreviation: AP, anteroposterior. 
Novice sonographers should start with scanning the aorta in the axial plane. The transducer marker should be pointed to the patient's right. The aorta will be located anterior to the vertebral body of the spine. The vertebral body appears as a curved, hyperechoic structure with posterior shadowing. The IVC will be located to the anatomic right of aorta unless the transducer is inadvertently pointing to the patient's left side or the patient has an anatomic variant resulting in a left-sided IVC. Common causes of left-sided IVC include cardinal vein anomalies that occur during embryogenesis. This results in a duplicated IVC or left-sided IVC, which can be partial or complete. Less common causes of left-sided IVC include congenital cardiac defects and situs inversus. ${ }^{33}$ The transducer should then slowly be rotated clockwise until the sagittal plane of the aorta is visualized.

Paraaortic nodes are located around the aorta. Most large nodes are located anteriorly. However, some are located posteriorly and may displace the aorta anteriorly, away from the vertebral body. These nodes can be mistaken for the aorta due to its location and appearance. The two can be distinguished by using color flow Doppler, as the lymph nodes will not have intraluminal flow. ${ }^{26,34}$ Occasionally, the interface between lymph nodes and aorta is difficult to distinguish, resulting in a falsely enlarged aorta.

Novice sonographers who obtain an image of the aorta on a thin patient may inadvertently misidentify the spinal canal as the aorta if the image obtained on the monitor is at an inappropriate depth (Figure 5). Knowledge of anatomy and adjusting for appropriate depth will prevent this operator error.

\section{Difficulty visualizing aorta}

Excess bowel gas and obesity are two common barriers to obtaining a clear ultrasound image of the aorta. Bowel gas can be displaced from the area of interest with the application of gentle, continuous pressure on the abdomen. The patient may need to be placed in the lateral decubitus position with concomitant gentle pressure on the abdomen to displace bowel to the side. . $^{2635,36}$

Increased body mass index is an obstacle for optimal ultrasound image acquisition. Increased waist circumference and variability in ultrasound measurements of the abdominal aorta have been reported. ${ }^{37}$ A low frequency $2-5 \mathrm{MHz}$ curvilinear transducer is recommended for imaging aorta. Occasionally, the frequency will need to be decreased further in order to improve penetration. ${ }^{35}$

The presence of a ruptured AAA may make the tubular structure of the aorta wall difficult to identify. ${ }^{8,38}$ Ultrasonography has limitations in the detection of a ruptured AAA, since retroperitoneal fluid is poorly visualized with abdominal sonography. While robust evidence is lacking in support of the US diagnosis of AAA rupture, there have been some reported US findings that aid in the identification of ruptured AAA. A retroperitoneal hematoma, appearing as a pseudomass of variable echogenicity, can be visualized adjacent to the aorta in the TR axis. ${ }^{39}$ Other ultrasound findings suggestive of a ruptured AAA may include echogenic retroperitoneal fluid collection, AAA deformation with irregularity or the aneurysm morphology, luminal thrombus inhomogeneity, luminal thrombus interruption, floating thrombus attached on one side of the aorta lumen and free floating on the other side and a distinct, focal discontinuity of the outer wall of the aneurysm with active leakage of blood verified with color Doppler. ${ }^{39,40}$ While the focused assessment with sonography in trauma exam is often utilized as an adjunct to evaluate for free fluid in the abdomen, this is dependent on the presence of fluid in the peritoneal space, and the incidence of a positive focused assessment with sonography in trauma for AAA rupture is low. ${ }^{22}$ Ultrasonography is still the diagnostic modality of choice to evaluate the unstable patient, since it is

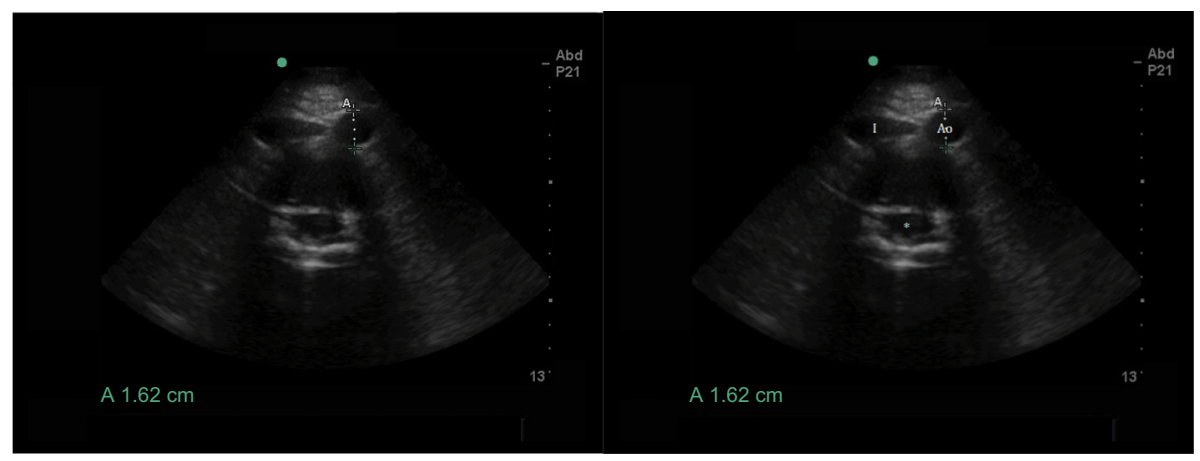

Figure 5 Axial ultrasound image of the abdominal aorta with inappropriate depth on the monitor where the spinal canal $(*)$ is visualized on the center of the screen. Notes: The aorta is correctly identified and measured in the near field.

Abbreviations: Ao, abdominal aorta; I, inferior vena cava. 
portable, quick and readily available at the bedside. Regardless, it is paramount to know the limitations of ultrasound, as patients may require emergent surgery based on clinical acumen. Diagnostic tests such as ultrasound may aid in the clinical evaluation of the patient, but do not replace clinical judgment. ${ }^{22}$ Patients may benefit from $\mathrm{CT}$ if they are hemodynamically stable, as CT can more accurately identify active bleeding and identify the breach in the aneurysmal wall and influence the surgical approach and technique.

\section{Level of experience}

Multiple studies have reported that various nonradiologists from a variety of subspecialties and different levels of training can identify the aorta with ultrasound with a relatively high degree of accuracy. ${ }^{6,41,42}$ Singh et al assessed the variability of sonographic measurements at different levels of the abdominal aorta and concluded that inexperienced sonographers might achieve acceptable performance with appropriate training and surveillance. ${ }^{43}$ Nguyen et al also further confirmed that novices could be trained to screen after 15 days. In the setting of technically difficult cases, continued quality improvement with scan experience was essential. ${ }^{44}$ Hoffmann et al suggest that credentialed Emergency Medicine sonographers with $<3$ years of experience were less likely to identify AAA in asymptomatic patients, compared to experienced colleagues. ${ }^{14}$ Emergency Physicians have demonstrated that an AAA can be identified with $100 \%$ accuracy after a 3-day course. ${ }^{6}$

In summary, a variety of errors and pitfalls can occur in the acquisition and interpretation of ultrasound imaging of the abdominal aorta. Further studies are warranted to assess the accuracy of the various techniques to measure the diameter of aorta. The OTO measurement of the AP aorta diameter is deemed less reproducible compared to the ITI approach. However, the OTO approach will less likely exclude AAA patients from screening and surveillance programs. Misdiagnosis errors can be avoided with improved knowledge and recognition of normal and pathologic anatomy. Obesity and bowel gas are common hurdles to optimal sonographic image acquisition of the abdominal aorta. Implementation of lower transducer frequency, changes in patient positioning and the application of gentle pressure may improve the ultrasound image. Ultrasound imaging is highly operator dependent. However, previous studies have demonstrated that sonographers with minimal experience are able to accurately identify AAA.

\section{Disclosure}

The authors report no conflicts of interest in this work.

\section{References}

1. AIUM Practice Guideline for the Performance of Diagnostic and Screening Ultrasound Examinations of the Abdominal Aorta in Adults. J Ultrasound Med. 2015;34(8):1-6.

2. Lewiss RE, Egan DJ, Shreves A. Vascular abdominal emergencies. Emerg Med Clin North Am. 2011;29(2):253-272.

3. Kent K. Clinical practice. Abdominal aortic aneurysms. $N$ Engl J Med. 2014;371(22):2101-2108

4. Brewster DC, Cronenwett JL, Hallett JW Jr, Johnston KW, Krupski WC, Matsumura JS; Joint Council of the American Association for Vascular Surgery and Society for Vascular Surgery. Guidelines for the treatment of abdominal aortic aneurysms. Report of a subcommittee of the Joint Council of the American Association for Vascular Surgery and Society for Vascular Surgery. J Vasc Surg. 2003;37(5):1106-1117.

5. LeFevre ML; U.S. Preventive Services Task Force. Screening for abdominal aortic aneurysm: U.S. Preventive Services Task Force recommendation statement. Ann Intern Med. 2014;161(4):281-290.

6. Kuhn M, Bonnin RL, Davey MJ, Rowland JL, Langlois SL. Emergency department ultrasound scanning for abdominal aortic aneurysm: accessible, accurate, and advantageous. Ann Emerg Med. 2000;36(3): 219-223.

7. Bentz S, Jones J. Towards evidence-based emergency medicine: best BETs from the Manchester Royal Infirmary. Accuracy of emergency department ultrasound scanning in detecting abdominal aortic aneurysm. Emerg Med J. 2006;23(10):803-804.

8. Rubano E, Mehta N, Caputo W, Paladino L, Sinert R. Systematic review: emergency department bedside ultrasonography for diagnosing suspected abdominal aortic aneurysm. Academic Emerg Med. 2013;20(2):128-138.

9. Beales L, Wolstenhulme S, Evans JA, West R, Scott DJ. Reproducibility of ultrasound measurement of the abdominal aorta. Br J Surg. 2011;98(11):1517-1525.

10. Ellis M, Powell JT, Greenhalgh RM. Limitations of ultrasonography in surveillance of small abdominal aortic aneurysms. Br J Surg. 1991;78(5):614-616.

11. Gurtelschmid M, Bjorck M, Wanhainen A. Comparison of three ultrasound methods of measuring the diameter of the abdominal aorta. $\mathrm{Br}$ J Surg. 2014;101(6):633-636.

12. Hartshorne TC, McCollum CN, Earnshaw JJ, Morris J, Nasim A. Ultrasound measurement of aortic diameter in a national screening programme. Eur J Vasc Endovasc Surg. 2011;42(2):195-199.

13. Thapar A, Cheal D, Hopkins T, Ward S, Shaloub J, Yusuf SW. Internal or external wall diameter for abdominal aortic aneurysm screening? Ann R Coll Surg Engl. 2010;92(6):503-505.

14. Hoffmann B, Bessman ES, Um P, Ding R, McCarthy ML. Successful sonographic visualisation of the abdominal aorta differs significantly among a diverse group of credentialed emergency department providers. Emerg Med J. 2011;28(6):472-476.

15. NHS Abdominal Aortic Aneurysm Programme News. http://aaa.screening.nhs.uk. Available from: Accessed June 17, 2017

16. Thompson SG, Ashton H, Gao L, Buxton MJ, Scott RA; Multicentre Aneurysm Screening Study (MASS) Group. Final follow-up of the Multicentre Aneurysm Screening Study (MASS) randomized trial of abdoinal aortic aneurysm screening. Br J Surg. 2012;99(12):1649-1656.

17. Chiu KW, Ling L, Tripathi V, Ahmed M, Shrivastava V. Ultrasound measurement for abdominal aortic aneurysm screening: a direct comparison of the three leading methods. Eur J Vasc Endovasc Surg 2014;47(4):367-373.

18. Martin K, Ramnarine K. Physics. Diagnostic Ultrasound Physics and Equipment. 2nd ed. Cambridge: Cambridge University Press; 2010

19. Meecham L, Evans R, Buxton P, et al. Abdominal aortic aneurysm diameters: a study on the discrepancy between inner to inner and outer to outer measurements. Eur J Vasc Endovasc Surg. 2015;49(1):28-32.

20. Powell JT, Brown LC, Forbes JF et al. Final 12-year follow-up of surgery versus surveillance in the UK Small Aneurysm Trial. Br J Surg. 2007;94(6):702-708. 
21. Fleming C, Whitlock EP, Beil TL, Lederle FA. Screening for abdominal aortic aneurysm: a best-evidence systematic review for the U.S. Preventive Services Task Force. Ann Int Med. 2005;142(3):203-211.

22. ACEP Emergency Ultrasound Imaging Criteria Compendium. Ann Emerg Med. 2016;68(1):e11-e48.

23. Muzaffar R, Kudva G, Nguyen NC, Osman MM. Incidental diagnosis of thrombus within an aneurysm on 18F-FDG PET/CT: frequency in 926 patients. J Nucl Med. 2011;52(9):1408-1411.

24. Anderson JC, Baltaxe HA, Wolf GL. Inability to show clot: one limitation of ultrasonography of the abdominal aorta. Radiology. 1979;132(3):693-696.

25. Ihara T, Komori K, Yamamoto K, Kobayashi M, Banno H, Kodama A. Three-dimensional workstation is useful for measuring the correct size of abdominal aortic aneurysm diameters. Ann Vasc Surg. 2013;27(2):154-161.

26. Reardon RF, Clinton ME, Madore F, Cook TP. Abdominal aortic aneurysm. In: Ma O, Mateer JR, Reardon RF, Joing SA, editors. Ma and Mateer's Emergency Ultrasound. 3rd ed. New York, NY: McGraw-Hill; 2014.

27. Baird IM. Saccular aneurysms of the abdominal aorta: report of three cases and review. AMA Arch Intern Med. 1953;91(5):626-632.

28. Shang EK, Nathan DP, Boonn WW, et al. A modern experience with saccular aortic aneurysms. J Vasc Surg. 2013;57(1):84-88.

29. Kristmundsson T, Dias N, Resch T, Sonesson B. Morphology of small abdominal aortic aneurysms should be considered before continued ultrasound surveillance. Ann Vasc Surg. 2016;31:18-22.

30. Taylor BV, Kalman PG. Saccular aortic aneurysms. Ann Vasc Surg. 1999;13(6):555-559.

31. McPhee JT, Hill JS, Eslami MH. The impact of gender on presentation, therapy, and mortality of abdominal aortic aneurysm in the United States, 2001-2004. J Vasc Surg. 2007;45(5):891-899.

32. Forbes TL, Lawlor D, DeRose G, Harris KA. Gender differences in relative dilatation of abdominal aortic aneurysms. Ann Vasc Surg. 2006;20(5):564-568.
33. Spentzouris G, Zandian A, Cesmebasi A, et al. The clinical anatomy of the inferior vena cava: a review of common congenital anomalies and considerations for clinicians. Clin Anat. 2014;27(8):1234-1243.

34. Bieker T. Midabdominal mass: possible ascites. In: Sanders RC, Winter T, editors. Clinical Sonography: A Practical Guide. 4th ed. Philadelphia, PA: Lippincott, Williams \& Wilkins; 2006.

35. Schaberle W, Leyerer L, Schierling W, Pfister K. Ultrasound diagnostics of the abdominal aorta: English version. Gefasschirurgie. 2015;20 (Suppl 1):22-27.

36. Cosford PA, Leng GC. Screening for abdominal aortic aneurysm. Cochrane Database Sys Rev. 2007;2:CD002945.

37. Pleumeekers HJ, Hoes AW, Mulder PG et al. Differences in observer variability of ultrasound measurements of the proximal and distal aorta. J Med Screen. 1998;5(2):104-108.

38. Lederle FA. In the clinic. Abdominal aortic aneurysm. Ann Intern Med. 2009;150(9):ITC5-1-15; quiz ITC5-16.

39. Catalano O, Siani A. Ruptured abdominal aortic aneurysm: categorization of sonographic findings and report of 3 new signs. J Ultrasound Med. 2005;24(8):1077-1083.

40. Bhatt S, Ghazale H, Dogra VS. Sonographic evaluation of the abdominal aorta. Ultrasound Clin. 2007;2(3):437-453.

41. Lin PH, Bush RL, McCoy SA, et al. A prospective study of a handheld ultrasound device in abdominal aortic aneurysm evaluation. Am J Surg. 2003;186(5):455-459.

42. Riegert-Johnson DL, Bruce CJ, Montori VM, Cook RJ, Spittell PC. Residents can be trained to detect abdominal aortic aneurysms using personal ultrasound imagers: a pilot study. J Am Soc Echocardiogr. 2005;18(5):394-397.

43. Singh K, Bonaa KH, Solberg S, Sorlie DG, Bjork L. Intra- and interobserver variability in ultrasound measurements of abdominal aortic diameter. The Tromso Study. Eur JVasc Endovasc Surg. 1998;15(6):497-504.

44. Nguyen AT, Hill G, Versteeg MP, Thomson IA, van Rij AM. Novices may be trained to screen for abdominal aortic aneurysms using ultrasound. Cardiovasc Ultrasound. 2013;11:42.
Journal of Vascular Diagnostics and Interventions

\section{Publish your work in this journal}

The Journal of Vascular Diagnostics and Interventions is an international, peer-reviewed journal of diagnostics, focusing on non invasive vascular investigation methods involved in the evaluation of vascular diseases. The journal is committed to the rapid publication in the fields of vascular diseases. Original research, review, case reports, expert

\section{Dovepress}

opinion and commentaries are all considered for publication. The manuscript management system is completely online and includes a very quick and fair peer-review system, which is all easy to use. Visit http://www.dovepress.com/testimonials.php to read real quotes from published authors. 